See discussions, stats, and author profiles for this publication at: https://www.researchgate.net/publication/339899348

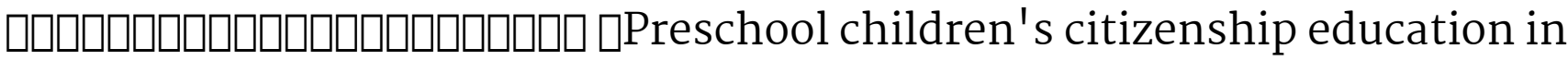
global context: the transformation of ideas and development trends $\square$

Article $\cdot$ November 2019

CITATION

1

1 author:

Q Peng $X u$

Victoria University of Wellington

6 PUBLICATIONS 1 CITATION

SEE PROFILE
READS

24

Some of the authors of this publication are also working on these related projects:

Project Discourses of citizenship in early childhood settings: A cross-national perspective in the global context View project 


\section{国际视域下学前儿童公民教育: 理念嬗变与发展趋势}

徐鹏

( 惠灵顿维多利亚大学教育学院, 新西兰惠灵顿 6012)

[摘要] 近年来, 学前儿童公民教育愈发受到各国学者的关注, 并逐渐成为学前教育 课程的重要内容，传统的儿童公民理念也出现了新的发展动向。首先，作为儿童公民教育 的核心，儿童公民身份正在由“等待中的公民”向“今日之公民”逐渐过渡；其次，儿童 公民身份的核心范畴也由单纯包含儿童权利扩展为主动参与、身份认同和归属感、公民责 任等内容。上述理念嬗变彰显了学前公民教育丰富公民理念与内涵、以公平为逻辑起点、 关注儿童参与和体验、立足本土历史文化等发展趋势。但是, 学前儿童公民教育的未来发 展仍然面临诸多潜在挑战, 如理念的转向困难、本土立场难以明确以及课程内容与实践的 形式化等。

[关键词］学前儿童；公民教育；公民身份

中图分类号: G619.1 文献标识码: A 文章编号: 1003-7667 (2019) 11-0096-09

当下，在西方民主国家中，青少年的政治参 与热情、履行公民权责的兴趣逐渐下降。为了扭 转这种趋势，进而更好地促进国家的民主建设， 公民教育愈发受到西方各国的重视。作为儿童 终身学习和发展的奠基阶段，学前教育阶段的 公民教育也逐渐成为西方国家学前教育课程的 重要内容。经济合作与发展组织（OECD）《强 力开端 2017: 早期儿童教育和看护关键指标》

( Starting Strong 2017: Key OECD Indicators on Early Childhood Education and Care ) 的研究报 告显示，2011 年在学前教育阶段开展公民教育 的国家数量不足 20 个, 到了 2015 年, 该数字则 上升到大约 80 个。 ${ }^{[1]}$ 此外, 近年的研究表明, 学 前教育阶段的公民教育对儿童的后继学习和全 面发展具有不可替代的奠基性作用。公民教育
不但满足了学龄前儿童个性化的教育需求，帮 助他们获得关于文化、民族的身份认同感 ; ${ }^{[2]}$ 而 且能够帮助儿童形成和提升尚处于萌芽阶段的 对于权利、责任、参与、平等等概念的认识，为日 后更好地参与到政治、经济和社会生活之中奠 定基础。 ${ }^{[3]}$ 在此背景之下，本文将着重分析近年 来西方学界关于学前儿童公民的理念嬗变, 进 一步分析其显著特征和发展趋势，思考学前儿 童公民教育所面临的潜在挑战。

\section{一、学前儿童公民教育的理念嬗变}

公民身份（citizenship）是公民教育的核心。 ${ }^{[4]}$ 理解和界定儿童的公民身份、梳理和概括其所囊 括的核心范畴及主要内容, 既是我们梳理国际上 关于儿童公民教育研究的起点, 又是我们全面理

(1)本文系北京市教育科学规划2018年度立项资助课题 “家园共育视域下儿童社会情绪学习（SEL）的课程建构与实践优 化研究”（项目编号：CCEA18143) 的阶段性研究成果。

作者简介：徐鹏，男，新西兰惠灵顿维多利亚大学教育学院博士研究生。 
解儿童公民教育理论与实践的关键所在。

\section{（一）学前儿童的公民身份}

当下西方学界关于 “儿童公民” 的概念界 定尚存在许多争议，不同的理论流派对于儿童 公民存在着不同的界定方式。总的来看，当下西 方学界对儿童公民身份的界定正由 “等待中的 公民”向“今日之公民”过渡。

\section{1. 等待中的公民}

传统的公民共和主义（ civic republican）和 自由主义 ( liberalism) 将儿童视为 “等待中的 公民”（ citizens in waiting），原因在于儿童的发 展水平低于成人, 缺乏行使公民权利以及履行 公民义务的能力。 ${ }^{[5]}$ 自由主义以权利为核心，认 为公民权利、政治权利和社会权利是构成公民 身份最重要的维度 ; 共和主义以公共责任为基 础, 认为纳税、服兵役、参与政治活动是每一个 公民必须履行的义务。 ${ }^{[6]}$ 相较于成人，儿童既没 有行使权力的能力, 亦无法胜任公民所应承担 之义务，因此只能被视为 “等待中的公民”。对 儿童教育的主要目的是培养未来的合格公民， 在学前教育阶段也并未设置与公民教育相关的 课程内容。以英格兰 20 世纪末基于公民共和主 义的公民教育改革为例, 公民教育开始于初等 教育末期，涵盖关键阶段3（11～14岁）和关键 阶段4（14１6岁），其主要的教育内容是有关 国家政治体制、公民权利和责任等内容。与此同 时，随着新自由主义（neoliberalism）的兴起，以 美国、加拿大等西方国家为主导的学前教育改 革逐渐呈现出注重管理绩效、关注课程质量、将 儿童视为经济发展的人力资本等倾向。在新自 由主义影响下, 公民教育的相关内容开始进人 学前教育阶段，儿童公民观由传统的 “等待中的 公民” 演化为 “未来的工人” ( citizen-workers of the future ）。以美国为例, 基于新自由主义教育 改革, 学前教育以儿童的人学准备为主要目标, 儿童并未被视为公民，虽然儿童被视为一种可 能产生丰厚回报的 “投资品” ，但为了培养能够 胜任后继学习和工作的劳动力, 其课程标准中 设立了与公民教育相关的领域内容。在美国早 期教育课程标准《开端计划早期学习成果框架》

( Head Start Early Learning Outcomes framework)
中, 与儿童公民教育相关的合作、参与等内容就 包含在新提出的社会情绪发展和学习品质等领 域之中。但是, 由于过分强调与未来工作相关的 核心素养和关键技能培养，过于重视遵守规则 的意识的培养，儿童的全面发展、主动参与和质 疑的批判能力、身份认同及归属感、对多元文化 的理解和认同等在很大程度上被忽视了。

\section{2. 今日之公民}

伴随着传统公民教育理念在儿童公民教育 领域弊端的日益凸显, 同时伴随着 20 世纪 90 年 代初的 “儿童运动” ，以及批判主义、跨国主义、 多元文化主义等理论流派的发展, “今日之公 民”（ citizens now）的儿童公民观逐渐得到了世 界各国学者的认同。以批判主义教育学为例, 其 所倡导的 “反歧视课程” (anti-bias curriculum) 就强调了儿童的公民身份，并倡导公民教育应 该帮助儿童建立自信和身份认同，培养多元文 化认同感, 发展批判性思维。而总的来看, “今日 之公民” 的儿童公民观对于学龄前儿童的公民 教育有如下几点影响 : 首先, 这种儿童公民观颠 覆了传统的 “等待中的公民” 的儿童公民身份界 定, 进一步丰富了学前儿童公民教育的内涵, 使 公平、平等、多元化等价值理念成为公民教育的 重要内容; 其次, 儿童公民身份由传统意义上的 法律地位和社会关系延伸为早期教育阶段的经 验与实践。 ${ }^{[8][9]}$ 这也使得公民教育由单纯的知识 传授和价值观养成转变为注重儿童的参与和体 验, 以体验式学习 (experience learning) 为基础 的教学方式也取代传统的直接教学方式, 成为 培养儿童公民素养和能力的基本途径之一。当 下, 越来越多的研究肯定了儿童具有作为一名 公民所应具有的关于权利和义务的意识以及主 动参与的能力，同时也批评了传统的 “等待中的 公民” 的儿童公民观，认为它在教育理念上忽视 了儿童的权利和主体性, 在教育实践中忽视了 儿童的主动参与和实际体验。例如, 英格兰学者 奥司勒（Audrey Osler）和斯塔基（Hugh Starkey） 就认为，在不正视儿童公民身份的前提下，儿童 在实际的教学过程中会感觉到被排除在了学校 和社区之外, 这将不利于他们身份的认同和归 属感的养成。 ${ }^{[10]}$ 阿黛尔（Jennifer Adair）等学者 
在一个关于美国、澳大利亚、新西兰学前儿童公 民教育的比较研究中也指出, 儿童并非 “等待中 的公民” , 在日常的生活和学习中, 他们已经具 有公民的意识和行为, 能够关心集体, 并能够为 集体做出贡献。[11]

综上所述，儿童公民身份因社会、政治和文 化背景而变化, 新兴理论对于传统理论的挑战 和批判并非在于否定其对于儿童公民身份及公
民教育的贡献。与之相反，在尊重儿童权利和儿 童主体性的基础之上，批判主义等新兴理论批 判性地接受并丰富了传统公民理论中关于公民 参与、权利、个体发展等内容，同时倡导多元化 的培养内容、多样化的培养方式和差异化的公 民身份，从而逐渐形成了一种更加包容的、多元 的、适宜的学前儿童公民身份界定方式，也丰富 了学前儿童公民的核心范畴（详见表1）。

表1. 学前儿童公民身份界定及相应理论流派观点比较

\begin{tabular}{|c|c|c|c|c|c|c|}
\hline 儿童公民身份 & \multicolumn{4}{|c|}{ 等待中的公民 } & \multicolumn{2}{|c|}{ 今日之公民 } \\
\hline 理论流派 & 共和主义 & 自由主义 & 新自由主义 & 批判主义 & 跨国主义 & 多元文化主义 \\
\hline 主要观点 & $\begin{array}{l}\text { 1. 重视公民的社 } \\
\text { 会责任及贡献 } \\
\text { 2.强调公民参与 } \\
\text { 3. 关注集体利益 }\end{array}$ & $\begin{array}{l}\text { 1.尊重个人权利 } \\
\text { 2.将公民视为一 } \\
\text { 种身份 }\end{array}$ & $\begin{array}{l}\text { 1.重视学前教育 } \\
\text { 质量 } \\
\text { 2.制定课程标准 } \\
\text { 和质量评价标准 } \\
\text { 3.重视核心素养 } \\
\text { 的培养 }\end{array}$ & $\begin{array}{l}\text { 1.尊重儿童多元 } \\
\text { 化的社会文化背 } \\
\text { 景 } \\
\text { 2.关注社会公平 } \\
\text { 3.重视儿童经验 } \\
\text { 4.倡导 “做中学” }\end{array}$ & $\begin{array}{l}\text { 1.倡导多维度的 } \\
\text { 公民身份（例 } \\
\text { 如，国家公民、 } \\
\text { 世界公民 ) } \\
\text { 2.强调平等、民 } \\
\text { 主等理念 }\end{array}$ & $\begin{array}{l}\text { 1.重视少数族裔 } \\
\text { 的权利 } \\
\text { 2.重视少数族群 } \\
\text { 的文化 } \\
\text { 3.重视对于文化 } \\
\text { 的同感 }\end{array}$ \\
\hline
\end{tabular}

主要资料来源 : Kathleen Knight Abowitz, Jason Harnish. Contemporary Discourses of Citizenship:[J]. Review of Educational Research, 2006,76(4):653-690. Lois Christensen, Jerry Aldridge. Critical Pedagogy for Early Childhood and Elementary Educators[M]. Springer Netherlands, 2013.

\section{（二）学前儿童公民身份的核心范畴}

儿童公民身份的演变逐渐丰富了学前儿 童公民的核心范畴，进而为在学前阶段开展公 民教育提供了内容依据。1989年发布的《儿童 权利公约》使儿童权利话语成为儿童公民教 育的基础。近十年来, 西方学者在尊重儿童权 利的基础上，对学前儿童公民所包含的核心范 畴进行了深人的探讨。李斯特（Ruth Lister）指 出，一个全面的关于儿童公民的定义需要包含 身份、权利、责任、平等的地位以及尊重和认可 （equality of status, respect and recognition）等 四个方面。 ${ }^{[12]}$ 菲利普斯 (Louise Phillips) 和莫 洛尼（Kerryn Moroney）则认为，儿童公民的核 心范畴包含公民身份、集体责任、公民主体性、 公民意识和公民参与等五个方面。 ${ }^{[13]}$ 总的来 看, 西方学界关于学前儿童公民核心范畴的探 讨主要涵盖以下四个方面。

\section{1. 儿童权利}

儿童权利是将儿童视为 “今日之公民” 的 逻辑起点，也是构筑现代儿童公民身份的基石。 《儿童权利公约》为不同年龄、性别和民族的儿
童设立了权利的基本标准和原则。相关学者基 于该公约内容, 进一步明确了学龄前儿童的权 利。例如，史密斯（AnneSmith）认为，学龄前儿 童的公民权利主要包含参与权、被保护权、接 受教育和看护权。 ${ }^{[14]}$ 当下西方学界的相关研究 指出, 儿童权利的真正落实和普及既需要在国 家政策层面明确和强调儿童的公民权利，同时 也需要在实践层面帮助儿童了解权利内涵、养 成权利意识、逐渐学会尊重和维护自身及他人 权利。例如, 加拿大阿尔伯塔省早期教育课程 标准《游戏、参与和可能性 : 阿尔伯塔早期学习 和儿童看护课程框架》（Play, Participation, and Possibilities: an Early Learning and Child Care Curriculum Framework for Alberta ）提出了 “民 主行为” 这一学习目标, 强调儿童需要学会理解 自身和他人的权利。在课程实践中，教师可以鼓 励和支持儿童参与到游戏和学习活动的计划过 程, 并为儿童提供发表自己意见和参与决策的 机会。 ${ }^{[15]}$

\section{2. 主动参与}

参与是儿童的基本权利之一。《儿童权利公 
约》指出, 对于所有能够影响儿童人生发展的 事情和活动，儿童都享有积极参与的权利。近年 来许多研究将参与和权利并列为儿童公民的核 心范畴之一, 并指出参与并非儿童后天习得, 而 是一种出于天性的、追求和实现社群利益的公 民行为。 ${ }^{[16]}$ 在学前教育阶段, 儿童逐渐建构了对 于自己、他人和世界的认识, 并开始意识到自己 具有发起和参与各种事情的权利和能力, 鼓励 儿童的参与可以帮助他们养成民主意识, 并培 养其作为公民的能力和素养。尽管当下儿童参 与仍然是一个研究难点, 但是许多国家依然将 参与作为了学前教育阶段课程的重要内容。以 澳大利亚为例, 其早期教育课程标准《归属、存 在和形成：澳大利亚幼儿教育框架》（Belong, Being and Becoming: the Early Years Learning Framework for Australia，下文简称 《幼儿教育框 架》) 就强调了社区参与对于儿童公民教育的重 要性, 并建议教师鼓励儿童积极参与社区的活 动, 尝试为社区做出力所能及的贡献。[17]

\section{3. 身份认同和归属感}

儿童公民的第三个核心内容是身份认同和 归属感。在学前教育阶段, 儿童已经开始意识到 自身与他人的异同之处，进而形成对于自我身 份的认同。肯普 (Kristen Kemple) 通过比较社会 素养和公民素养后发现, 公民身份认同是儿童 发展其他核心素养的基础，基于对自身的认同， 儿童才能够在设定目标的时候进行自我导向并 坚持完成既定目标，逐步发展同理心、沟通等奠 基未来学习和社会生活的关键能力。 ${ }^{[18]}$ 此外，儿 童在小组及社区活动中的参与和贡献也基于 他们已经形成的对于文化、社会、宗教等的认同 和归属感。新西兰、加拿大等国家均将身份认同 和归属感作为学前教育课程的重要内容。如, 加 拿大不列颠哥伦比亚省的《归属、反思、多样性 和参与 : 早期学习框架》 (Belonging, Reflection, Diversity and Engagement: Early Learning Framework）就将归属感和身份认同列为学龄前 儿童的发展目标之一。新西兰《特发瑞奇 : 早期 课程》 (下文简称《特发瑞奇》) 将归属感作为五 大教育领域之一, 并将儿童身份认同作为贯穿 五大教育领域的重要课程内容。以健康领域为
例, 该领域的发展需要教师营造一个安全、稳定 和能够响应儿童需求的环境, 同时这种环境也 能够更好地支持儿童形成对于自身和环境的认 同感。[19]

\section{4. 公民责任}

儿童公民的最后一个核心内容是责任。对 于学龄前儿童来说, 他们并不能够履行成人的 公民责任（如纳税、服兵役等）。然而, 这并不 能否认他们正在学习亦或是已经具备了一些 履行公民基本责任的意识、能力和素养。总的 来看, 西方学界对学前儿童责任的划分主要包 括自我照看、家庭责任、道德品质、环境责任、 学校责任、社区责任等方面。在可以观察到的 行为中, 学龄前儿童已经能够显示出分享、关 心他人感受等能力, 并能够维护公共区域的 秩序, 看护班级和学校的设备和玩具, 这些都 是儿童日后承担公民责任所必需的意识和能 力。对于教师和家长, 帮助儿童学会为自己的 言行负责任, 并能够了解和遵守基本的社会准 则和法律法规, 对儿童未来成为一名合格的 公民大有帮助。以美国华盛顿州《早期学习和 发展指南 : 从出生到 3 年级》 (Early Learning and Development Guidelines: Birth through 3rd Grade ）为例, 其 5 岁儿童的科学领域发展目标 就指出, 儿童需要承担起照顾身边动植物的 责任（喂鱼、浇花等）; 在该年龄段的社会学习 （social studies）领域同样也强调培养儿童对于 责任的认识, 主要的教学策略包含鼓励儿童在 角色扮演游戏中体验不同的家庭角色和社区 角色, 或者与他们讨论长大后所希望从事的职 业等。 ${ }^{[20]}$

\section{二、学前儿童公民教育的国际趋势}

学前儿童公民理念的变迁丰富了传统理论 流派对于公民概念的界定, 扩展了公民权利与责 任的范畴。学前教育阶段的公民教育逐步以公平 为逻辑起点, 教育方式由传统的课堂教学逐渐转 变为儿童参与和体验。与此同时, 一些国家和地 区开始基于本土的历史和文化建构了学前儿童 公民教育模式, 为突破传统的、以英美为主导的 公民教育话语体系带来了极大的可能性。 


\section{（一）扩展公民权责，丰富公民理念内涵}

传统意义上，公民是权责统一、个体主体 和类主体相统一的人, 没有无义务的权利, 也 没有无权利的义务。 ${ }^{[21]}$ 也正因此, 由于儿童, 尤其是学龄前儿童, 因为他们在发展和认知水 平上与青少年和成人处于不同阶段，普遍被认 为缺乏权责的意识和履行权责的能力, 因此 很长时间被排除在公民这一范畴之外。然而, 基于近年来西方学界在理论和实践方面的进

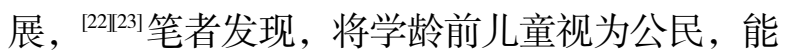
够使理论研究者和实践者重新审视和理解公 民教育的本体论，变革了传统意义上的局限于 成人的公民身份和公民教育，丰富了权利、义 务、民主等核心概念。首先，公民的权责不再局 限于成人公民的人身自由、选举投票、纳税等 内容，也囊括了儿童公民的受教育、从事与年 龄相宜的游戏和学习活动、以及初步了解并能 够遵守公共道德和社会准则等内容。其次, 将 儿童视为公民, 并非仅仅因为他们已经显示出 履行公民义务的素养和能力, 而是因为基于该 立场下的儿童教育能够变革成人对于儿童公 民身份的传统观念, 从而更好地培养他们在成 年以后履行成人公民义务的态度、价值观和能 力。以北欧国家为例, 其高水平的学前教育正 是基于该地区将儿童视为 “今日之公民”，由 此幼儿教育成为 “民主实践场” ${ }^{[24]}$ ，不但强调儿 童权利以及民主参与，而且鼓励儿童在参与的 过程中成为真正的公民。如哈斯（Chris Haas） 和阿什曼（Greg Ashman）指出, “在治愈世界 的伤口之前, 他们 (儿童) 需要首先学会热爱 这个世界” ${ }^{[25]}$ 。最后，儿童作为公民，能够为成 人带来审视权利、责任、公平等核心公民范畴 的全新视角。吉尔 (Judith Gill) 和霍华德（Sue Howard）通过研究发现, 尽管儿童并不具备足 够的关于公民、民主和政治的知识，但是他们 能够就日常生活中关于权利、社会发展等话 题进行讨论并给出建设性的意见。 ${ }^{[26]}$ 恩格勒

(Christina Ergler) 等人则通过对新西兰和澳大 利亚 $3 \sim 5$ 岁幼儿的调查, 证明学龄前儿童能够 为城市环境的设计提供不同于成人的有价值的 意见。 ${ }^{[27]}$

\section{（二）以公平为逻辑起点, 保障儿童权利的 实现}

1989年颁布的《儿童权利公约》明确了儿 童所应享有的权利，所有缔约国也均将儿童权 利作为了早期教育的理论基石。然而，长期以来 忽视儿童的公民身份和地位、忽视儿童公民参 与和体验等现象使得对儿童权利的尊重仅仅是 纸上谈兵，而没有真正地体现在教育理念和实 践之中。随着儿童公民理念逐渐受到认同，如何 进一步体现对于儿童权利的尊重, 凸显公平作 为公民教育价值逻辑起点的重要性，也成为当 下儿童公民教育的重要发展趋势之一。一直以 来，对儿童公民的忽视导致了儿童在公民教育 的过程中被排斥和边缘化。以公平作为儿童公 民教育的逻辑起点，能够为儿童得到同成人公 民平等的地位和待遇提供前提。此外，在正视儿 童公民身份的前提下，教师和家长能够与儿童 形成一种更加民主和平等的关系，营造一种更 为和谐与平等的园所环境和家庭氛围, 这能够 使儿童意识到自己被他人认可与重视，从而进 一步激发他们参与日常游戏和学习活动以及社 区活动的热情。最后，在平等对待的基础之上， 教育公平所提倡的差异对待和补偿原则能够使 来自不同文化背景的、处于不同发展水平的儿 童得到差异化的公民教育，实现每一名儿童的 全面发展和个性化发展，也能够使处境不利的 儿童得到重视，从而为他们日后成为主动的、负 有责任的社会公民奠定基础。

（三）注重儿童的参与体验，变革传统的公 民教育内容与实践

理念内涵的丰富也带来了教育内容的扩展 以及教育实践的变革。首先，基于对儿童主体性 和公民权利的重视，越来越多的国家将培养儿 童公民作为了学前阶段课程的主要目标之一。 以澳大利亚《幼儿教育框架》为例, 其主要目标 在于使儿童成为 “成功的学习者、充满自信与 创造力的个人以及积极知情的公民（active and informed citizens ) ” ${ }^{[28]}$ 。在具体的教学目标方面, 也囊括了身份认同感、形成对于周围环境的理 解并尝试做出贡献、有效沟通交流、尊重文化多 样性和民族多样性等与儿童公民教育紧密相关 
的内容。除此之外, 越来越多的学者也开始关注 游戏对学前儿童公民教育的作用。拉金斯（Cath Larkins）和阿黛尔等学者都指出，游戏能够支持 儿童的公民行为, 并为儿童提供了表达和体验 公民权利、公民身份的最佳机会。 ${ }^{[29[30]}$ 同时，核心 素养也逐渐与学前儿童的公民教育紧密联系在 一起, 为学前阶段的公民教育提供了课程指引。 在新西兰《特发瑞奇》课程中，健康领域强调儿 童需要能够管理和表达自己的想法和需要, 这 就与自我管理（managing self）这一核心素养紧 密联系。 ${ }^{[311}$ 加拿大安大略省《培养学前儿童的坚 韧性 : 0-6岁儿童家长手册》（Building Resilience in Young Children: Booklet for Parents of Children from Birth to Six Years ）则将儿童的责任与参与 作为了坚韧性等素养发展的重要影响因素, 指 出承担责任和积极参与是个体获得自我发展、

建立良好人际关系、为家庭和社区做出贡献的 途径和保障。 ${ }^{[32]}$ 此外，尽管社会学习被视为儿童 公民教育的主要课程领域, 但公民教育也与其 他发展领域或学科紧密联系、相互影响。对于儿 童公民知识、能力和素养的教育贯穿于各个课 程领域之中, 这样既确保了儿童各个领域的均 衡发展，同时也能够使儿童在学前阶段获得更 加全面的公民教育。科尔森（Caroline Cohrssen） 和佩吉（Jane Page）在一项针对儿童数学领域发 展的研究中发现, 鼓励儿童积极参与数学领域 的活动, 不仅能够锻炼儿童的数理逻辑思维, 而 且也能够帮助他们发展作为积极公民的核心素 养。 ${ }^{[33]}$

\section{（四）立足本土历史文化，突破传统英美公 民话语体系}

当下儿童公民理念和实践的变迁同样也体 现了世界各国对传统英美关于公民教育的主流 话语的挑战。 20 世纪 80 年代以来, 以英美为代 表的新自由主义改革不仅推动了国内经济的发 展, 而且也影响着世界各国经济以及教育发展 的走向。学前教育领域的改革也愈发关注学校 管理绩效、投资回报收益、教育质量与评价等方 面，并对其他各国政策话语和教育传统带来了 冲击。以北欧国家为例, 其传统的基于民主和儿 童幸福的社会教学法取向就受到了英美人学准
备取向的冲击。 ${ }^{[34]}$ 但是, 新自由主义注重市场逻 辑而忽视了教育的育人本质，重人学准备而忽 视了儿童的权利。对于儿童公民教育来说, 这种 人学准备取向的教育不但不利于他们认同、继 承和发扬本民族和本地区的优秀文化传统，而 且由于在教育过程中过分重视知识、能力的养 成而忽视儿童的直接经验与亲身体验, 从而无 法真正实现儿童的全面发展。同时，单纯借鉴西 方教育实践经验而忽视本国经济发展水平、社 会文化背景也会导致文化殖民现象的发生，不 利于国家公民的培养以及本土文化的传承和发 扬。国际上部分国家和地区也开始尝试建构不 同于主流英美话语体系的、适合于本地区情况 的公民教育课程。以苏格兰为例，虽然与英格兰 同属于英国，但是由于其不同的文化历史传统， 苏格兰地区的政策制定者和教育研究者就以建 构不同于英格兰的课程为出发点, 在培养 “苏格 兰公民” 的理念引领下建立了基于 “今日之公 民” 的《卓越课程》（Cumiculum for Excellence）。 该课程覆盖了学前教育、初等教育和中等教育 阶段, 并将培养主动和负责任的儿童公民作为 $3 \sim 16$ 岁儿童教育的主要目标之一。此外, 一些 国家也将国内少数民族或者土著民族的历史文 化传统作为早期公民教育重要的理论基础和 课程内容。例如, 加拿大政府于 2018 年出台了 《原住民儿童早期学习和看护框架》（Indigenous Early Learning and Child Care Framework）, 突出 了梅蒂斯（Metis）和因纽特（Inuit）等土著民族 的历史和文化价值, 为学前儿童的文化身份认 同、社区参与等公民能力和素养的培养提供了 重要参照。此外, 新西兰的《特发瑞奇》课程则 将毛利教育理论“卡帕帕毛利理论”（Kaupapa Maori Theory）和 “帕希菲卡方法”（Pasifika Approaches）作为培养儿童公民的理论基础。这 种理论和方法均强调将新西兰土著民族的传统 理念融人早期教育课程之中。以卡帕帕毛利理 论为例, 该理论基于毛利民族的历史文化传统, 强调儿童为平等的公民和主动的参与者, 倡导 将毛利文化中的传统价值理念，例如爱（aroha）、 赋权（mana）、团结（Kotahitanga）等作为培养儿 童公民的主要内容。 


\section{三、学前儿童公民教育未来发展的挑 战与思考}

虽然当下西方学界对于学前儿童公民身份 和公民教育的研究逐渐深人, 美国、加拿大、澳 大利亚、新西兰等国也逐步形成了具有一定可 操作性的学前儿童公民课程。但与此同时, 学前 阶段的公民教育仍然是各学段公民教育中最薄 弱的环节，面临着诸多的困难和挑战。

在理念层面, 学前儿童公民观的变革以及 不同公民理论体系的冲突与融合在为世界各国 教育改革提供新可能性的同时, 也带来了巨大 的挑战。如前所述，传统的 “等待中的公民” 的 儿童公民观忽视了儿童的主体性和权利, 限制 了学前儿童公民教育的内容和实践, 但因其扎 根于西方公民教育的传统理论之中, 因此仍具 有较大的影响力。笔者认为, 如果想要真正使 儿童权利不再只是纸上谈兵的话, 对于当下世 界各国的学前儿童公民教育发展, 其首要任务 是彻底转变 “无知” “慒懂” 等对儿童的传统印 象, 正视并重视他们的公民身份和参与权利, 形 成平等于成人但不同于成人的公民身份。前述 学者阿黛尔等人就认为, 如果社会和教育不能 够将儿童视为主动的贡献者的话, 最后教育出 的儿童仍然只能是无知和脆弱的。 ${ }^{[35]}$ 此外, 通过 分析近年西方学界的相关研究, 笔者发现, 单纯 借鉴其他国家的理念和模式, 并不适用于一个 国家学前儿童公民教育的发展和改革。在多种 理念的冲突融合中, 加拿大、新西兰等国家和苏 格兰等地区开始尝试摒弃美英等国的主流话语 和传统公民教育模式, 基于本国和本地区情境 建构适宜的儿童公民教育理念和模式。但如何 在国际主流话语和本土历史文化之间进行权衡 与取舍, 在培养儿童本土立场的同时兼具国际 化的视野, 仍然是各国未来公民教育发展的难 题。

在课程内容和实践方面, 如何将学前儿童 公民教育进一步系统化也面临诸多困难。在课 程内容中, 学前儿童公民教育课程囊括了公民 知识、态度、价值观、能力和素养等内容。但是, 如何明确平等、自由等民主价值, 平衡知识、能
力和素养的比重分配, 破解知识传授与素养养 成的二元对立, 都需要做进一步的研究。 ${ }^{[36]}$ 在课 程实施中, 当今的学前儿童公民教育摒弃了传 统的知识传授方式，而是基于学龄前儿童的发 展特点, 形成了一种以儿童为中心, 基于儿童 体验式学习, 重视儿童与周围人、事、物进行互 动的实践方式。但是，在成人与儿童的互动过 程中, 还存在着难以将儿童视为平等公民的困 难。例如, 一方面, 在儿童享有表达观点和被倾 听的权利的同时, 他们仍然需要被成人保护和 照顾 ; 另一方面，当下公民教育鼓励儿童主动参 与、大胆质疑, 但同时他们也需要服从权威、遵 守规则。根据迪瓦恩（Dympna Devine）等人的研

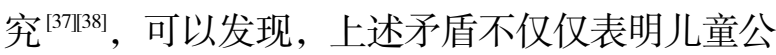
民理念尚处于冲突调和阶段，同时也体现了成 人公民与儿童公民在关系和互动上的权力失衡 （power imbalance）。这种失衡限制了成人对于儿 童公民身份的理解，使他们低估了学龄前儿童 公民教育的重要价值，难以在教育过程中组织 适宜儿童的民主参与和体验活动，也容易导致 在教育实践过程中儿童参与的形式化与公民体 验的表面化。基于已有研究, 笔者认为, 这种失 衡根源于长期以来的文化历史传统和教育实践 模式。真正扭转这种权力失衡, 则需要对学前儿 童公民教育进行更多跨学科的、多层次的、多视 角的持续性深人研究。

\section{四、结语}

加州大学洛杉矶分校的卡洛斯・托雷斯 （Carlos Torres）教授在2016年世界比较教育大 会的主旨报告《位于 21 世纪十字路口的比较教 育与世界比较教育学联合会》 (The State of the Art in Comparative Education and WCCES at a Crossroads in the 21st Century）中指出，民主、公 民身份和多元文化不仅应该是当下研究的理论 指导和方法论基础, 更应是研究者的热情所在, 这样才能够创造一个更美好、更具人文关怀、更 公正和可持续发展的世界。 ${ }^{[39}$ 当下西方学界虽 然已经在学前儿童公民教育方面取得了一些进 展, 并呈现出了积极的发展趋势, 但如何使儿童 能够真正成为建设这个美好世界的参与者和贡 
献者，仍然任重而道远。

\section{参考文献 :}

[1]OECD. Starting Strong 2017: Key OECD indicators on early childhood education and care[R]. Pairs: OECD Publishing,2017 : 132-133.

[2]Robin Elizabeth Hancock. Global Citizenship Education: Emancipatory Practice in a New York Preschool[J]. Journal of Research in Childhood Education, 2017,31(3):1-10.

[3]Joanne Lunn Brownlee, Laura Scholes, Sue Walker \& Eva Johansson. Critical values education in the early years: Alignment of teachers' personal epistemologies and practices for active citizenship[J]. Teaching \& Teacher Education, 2016,(59):261-273.

[4] 冯建军. 公民教育课程及其设计 $[\mathrm{J}]$. 东北师大学报 (哲 学社会科学版 ), 2015(1):9-14.

[5]Kathleen Knight Abowitz, Jason Harnish. Contemporary Discourses of Citizenship:[J]. Review of Educational Research, 2006,76(4):653-690.

[6][10]Audrey Osler, Hugh Starkey. Learning for Cosmopolitan Citizenship: Theoretical debates and young people's experiences[J]. Educational Review, 2003,55(3):243-254.

[7]Louis Phillips. Young children' s active citizenship: storytelling, stories, and social actions[D]. Queensland: Queensland University of Technology, 2010: 6.

[8][12]Ruth Lister. Why citizenship: Where, when and how children? Theoretical inquiries in Law, 2007,8(2):693-718.

[9]Louise Phillips. Possibilities and Quandaries for Young Children's Active Citizenship. Early Education and Development, 2011,22(5):778-794.

[11][30][35]Jennifer Keys Adair, Louise Phillips, Jenny Ritchie \& Shubhi Sachdeva. Civic action and play: examples from Maori, Aboriginal Australian and Latino communities[J]. Early Child Development \& Care, 2016,187(5-6):1-14.

[13][16]Louise Phillips, Kerryn Moroney. Civic action and learning with a community of Aboriginal Australian young children[J]. Australian Journal of Early Childhood, 2017,42(4):87-96.

[14]Anne Smith. Children as citizens and partners in strengthening communities[J]. American Journal of Orthopsychiatry, 2010,80(1):103-108.
[15]Alberta Ministry of Education. Play, participation, and possibilities: An early learning and child care curriculum framework for Alberta[EB/OL].(2015-02-10).[2018-12-23] http://childcareframework.com/play-participation-andpossibilities/.

[17][28]Department of Education. Belong, being \& becoming: the early years learning framework for Australia[EB/OL] .(2009-07-16)[2018-10-22]. https://docs.education.gov. au/node/2632.

[18]Kristen Kemple. Social Studies, Social Competence and Citizenship in Early Childhood Education: Developmental Principles Guide Appropriate Practice[J]. Early Childhood Education Journal, 2017(45)621-627.

[19][31]Ministry of Education.Te Whā riki. He Whā riki Matauranga mō ng ā Mokopuna o Aotearoa: Early Childhood Curriculum[EB/OL].(2017-04-12).[2018-0704]. https://education.govt.nz/assets/Documents/EarlyChildhood/Te-Whariki-Early-Childhood-Curriculum. pdf.

[20]Washington State Department of Early Learning. Early learning and development guidelines: birth through 3rd grade[EB/OL].(2012-01-01).[2018-09-10]. http://www. k12.wa.us/EarlyLearning/guidelines.aspx.

[21] 孙智昌. 公民教育的逻辑起点 [J]. 教育研 究 ,2011,32(11):13-17.

[22]Louis Phillips, Jenny Ritchie \& Jennifer Keys Adair. Young children' s citizenship membership and participation: comparing discourses in early childhood curricula of Australia, New Zealand and the United States[J]. Compare: A Journal of Comparative and International Education, 2018:1-23

[23]Caroline Bath, Rauni Karlsson. The ignored citizen: Young childrens subjectivities in Swedish and English early childhood education settings[J]. Childhood, 2016,23(4):554-565.

[24][34] 李敏谊, 郭宴欢, 陈肖琪. 北欧国家幼儿教 育和保育政策话语的新变迁 $[\mathrm{J}]$. 比较教育研究, 2018,40(5):89-97.

[25]Chris Haas, Greg Ashman. Kindergarten children's introduction to sustainability through transformative, experiential nature play[J]. Australasian Journal of Early 
Childhood, 2014,39(2):21-29.

[26]Judith Gill, Sue Howard. Knowing our place: children talking about power, identity and citizenship[J]. Pedagogijska Istraživanja, 2009,37(1):220-223.

[27]Christina Ergler, Kylie Smith, Cassandra Kotsanas \& Constance Hutchinson. What Makes a Good City in Preschoolers' Eyes? Findings from Participatory Planning Projects in Australia and New Zealand[J]. Journal of Urban Design, 2015,20(4):461-478.

[29]Cath Larkins. Enacting children' s citizenship: Developing understandings of how children enact themselves as citizens through actions and acts of citizenship $[\mathrm{J}]$. Childhood, 2014,21(1):7-21.

[32] 胡恒波, 霍力岩. 加拿大学前儿童坚韧性品质家庭 培养及启示一一以安大略省为例 $[\mathrm{J}]$. 比较教育研究, 2017,39(5):97-104.

[33]Caroline Cohrssen, Jane Page. Articulating a rights-based argument for mathematics teaching and learning in early childhood education[J]. Australasian Journal of Early Childhood, 2016,41(3):104-108.

[36]Bath, Caroline, Rauni Karlsson. The ignored citizen: Young children' s subjectivities in Swedish and English early childhood education settings.Childhood,2016,23(4): $554-565$

[37]Dympna Devine, Tom Cockburn. Theorizing children' s social citizenship: New welfare states and intergenerational justice. Childhood, 2018,25(2):142-157.

[38]Fiona Mayne, Christine Howitt \& Lé onie Rennie. A hierarchical model of children's research participation rights based on information, understanding, voice, and influence, European Early Childhood Education Research Journal, 2018,26(5): 644-656.

[39]Carlos Alberto Torres. The state of the art in comparative education and WCCES at a crossroads in the $21 \mathrm{st}$ Century[J]. RevistaLus ó fona de Educação, 2018 (41): $107-124$.

\title{
Preschool Children's Citizenship Education in Global Context: the Transformation of Ideas and Development Trends
}

\section{XU Peng}

(School of Education, Victoria University of Wellington, Wellington 6012, New Zealand)

\begin{abstract}
In recent years, preschool children's citizenship education has been emphasized globally and gradually becomes a main content in early childhood curriculum. Traditional ideas on early age citizens see some new changes. As the core of early age children's citizenship education, children's citizenship is gradually transferred from citizens in waiting to citizens today, including right, active participation, identity and sense of belonging, and collective responsibility. The above transformation highlights global trends such as enriching ideas and contents on citizenship, regarding equity as the logic starting point, paying attention to young children's participation and experience, and being rooted in indigenous context and culture. In the meantime, potential challenges still exist for the future development of young children's citizenship, such as the difficulty of transferring ideas, ambiguous standing point and the tokenism of curriculum content and practice.
\end{abstract}

Key words: preschool children; citizenship education; citizenship 